\title{
AVALIAÇÃO DE VIGAS DE MADEIRA LAMINADA COLADA DE CEDRINHO (Erisma uncinatum Warm.)
}

\author{
Pedro Gutemberg de Alcântara Segundinho ${ }^{1 *}$, André Luiz Zangiácomo², Marcelo Rodrigo Carreira ${ }^{3}$, \\ Antonio Alves Dias ${ }^{4}$, Francisco Antonio Rocco $\mathrm{Lahr}^{4}$
}

*Autor para correspondência: p_gutemberg2001@yahoo.com.br

RESUMO: A madeira laminada colada (MLC) é produzida a partir da colagem de peças de madeira. No Brasil, o emprego da madeira de espécies nativas para produzir elementos estruturais de MLC não ocorre em condições tecnológicas satisfatórias, sendo dificultado por não serem totalmente conhecidas as suas propriedades e seu desempenho em diferentes condições de serviço. Neste estudo, objetivou-se determinar e relacionar o módulo de elasticidade dinâmico $\left(\mathrm{E}_{\mathrm{Mvt}}\right)$ com o módulo de elasticidade estático $\left(\mathrm{E}_{\mathrm{M}}\right)$, o módulo de ruptura $\left(\mathrm{f}_{\mathrm{M}}\right)$, a resistência à compressão $\left(\sigma_{\mathrm{cp}}\right)$ e o módulo de elasticidade paralelo $\left(\mathrm{E}_{\mathrm{cp}}\right)$, e avaliar o desempenho da espécie nativa Cedrinho (Erisma uncinatum Warm.) na construção de vigas de MLC. As vigas de MLC foram produzidas com peças de madeira classificadas, coladas por adesivos à base de resorcina fenol formaldeído (RFF) e poliuretano (PUR), com intensidades de pressão de 0,8 MPa e 1,2 MPa e distribuições aleatórias e não aleatórias das lâminas. A avaliação dessas vigas de MLC também foi realizada por meio de corpos de prova de compressão paralela às linhas de cola e de delaminação. Verificou-se que o $\mathrm{E}_{\mathrm{Mvt}}$ obtido por meio de ensaios dinâmicos de vibração transversal apresentou um coeficiente de correlação com o $\mathrm{E}_{\mathrm{M}}$ obtido no ensaio de flexão estática $\left(\mathrm{R}^{2}=0,86\right)$ e significativo ao nível de $1 \%$ de probabilidade $(\mathrm{P}<0,01)$. Dessa forma, pode-se concluir que o $\mathrm{E}_{\mathrm{Mvt}}$ é um bom estimador do $\mathrm{E}_{\mathrm{M}}$. Constatou-se que essa espécie de madeira pode ser utilizada em MLC e encontra-se na classe de resistência $\mathrm{C} 30$ das folhosas.

Palavras-chave: Vibração transversal, vigas de MLC, madeira tropical, ensaios não destrutivos.

\section{EVALUATION OF GLUED LAMINATED TIMBER BEAMS OF CEDRINHO (Erisma uncinatum Warm.)}

\begin{abstract}
Glued laminated timber (glulam) is assembled layers of bonded pieces of timber. In Brazil, the use of native species timber to produce structural elements of glulam does not occur in satisfactory technological conditions. This is because the properties and performance of native species of wood, under various service conditions, are not entirely known. This study aims at determining and to relate the dynamic modulus of elasticity $\left(E_{M v t}\right)$ and the static modulus of elasticity $\left(E_{M}\right)$, the modulus of rupture $\left(f_{M}\right)$, the compression parallel strength $\left(\sigma_{c p}\right)$ and the parallel elastic modulus $\left(E_{c p}\right)$, and evaluating the performance of the native species Cedrinho (Erisma uncinatum Warm.) in the construction of glulam beams. Glulam beams were produced with random and nonrandom distributions of graded pieces of bonded lumber by using adhesives of phenol resorcinol formaldehyde (RFF) and polyurethane (PUR); the intensity of pressures used was $0.8 \mathrm{MPa}$ and $1.2 \mathrm{MPa}$. The evaluation of these glulam beams was also performed using samples of compression parallel to glue lines and delamination. It was found that the $E_{\text {Mvt }}$ obtained by transverse vibration dynamic testing revealed a correlation coefficient with the $E_{M}$ obtained on static bending test $\left(R^{2}=0.86\right)$ and significance level of $1 \%$ probability $(P<0,01)$. Thus, it can observed that the $E_{M v}$ is a good estimator of $E_{M}$. It was found that this wood species may be used for glulam, and that the hardwoods species is in strength class C30.
\end{abstract}

Key words: Transverse vibration, glulam beams, tropical timber, nondestructive testing.

\section{INTRODUÇÃO}

O emprego estrutural da madeira de espécies nativas do Brasil, proveniente de florestas certificadas, nem sempre ocorre em condições tecnológicas satisfatórias, apesar de sua versatilidade e de sua disponibilidade. Mesmo com esses aspectos favoráveis, seu uso é, às vezes, dificultado por não serem cabalmente conhecidas suas propriedades mecânicas e seu desempenho em diferentes condições de serviço. Conforme Instituto de Pesquisas Tecnológicas do Estado de São Paulo - IPT (2009), a utilização das florestas naturais ou plantadas, por meio de Projeto de Manejo Florestal aprovado pelo Instituto Brasileiro do Meio Ambiente e dos Recursos Naturais Renováveis (IBAMA), configura a forma correta de utilizar estes recursos naturais, porque parte do princípio de sustentabilidade, isto é, permite a recomposição da floresta de uma determinada área, viabilizando-a econômica, socialmente e ambientalmente.

\footnotetext{
${ }^{1}$ Universidade Federal do Espírito Santo - Jerônimo Monteiro, Espírito Santo, Brasil

${ }^{2}$ Universidade Federal de Lavras - Lavras, Minas Gerais, Brasil

${ }^{3}$ Universidade Tecnológica Federal do Paraná - Campo Mourão, Paraná, Brasil

${ }^{4}$ Universidade de São Paulo - São Carlos, São Paulo, Brasil
}

Cerne, Lavras, v. 19, n. 3, p. 441-449, jul./set. 2013 
A madeira laminada colada (MLC) é uma opção de uso, por se tratar de produto obtido pela associação de peças da madeira (lâminas) que requer precisão de fabricação em todos os seus estágios. As lâminas, unidas por colagem, ficam dispostas de modo que suas fibras estejam paralelas entre si (BODIG; JAYNE, 1993) e formem um componente estrutural. Tais lâminas de madeira são selecionadas, coladas com adesivo à prova d'água, sob pressão variável de 0,7 a 1,5 MPa. Estas atingem até $5 \mathrm{~cm}$ de espessura e podem ser emendadas por juntas em bisel ou dentadas, nas situações em que é necessário vencer grandes vãos (PFEIL; PFEIL, 2003). O aproveitamento proporcionado pela união de peças de pequenas dimensões para laminação de vigas estruturais é a maior vantagem da MLC. Portanto, trata-se de forma racional de emprego da madeira na construção de estruturas.

A MLC tem seus usos mais frequentes em estruturas de cobertura, elementos estruturais principais para pontes, torres de transmissão, edifícios, embarcações, entre outros. Isso se deve ao fato de adaptar-se a uma significativa variedade de formas e apresentar alta resistência a solicitações mecânicas, em função de seu peso próprio relativamente baixo (ZANGIÁCOMO; LAHR, 2007). Como uma das vantagens, permite a redução dos defeitos observados em peças de madeira maciça com grandes dimensões.

Segundo as prescrições da NBR 7190, da Associação Brasileira de Normas Técnicas - ABNT (1997), as espécies mais aconselhadas para o emprego em MLC tem valor de referência de densidade até 0,75 $\mathrm{g} / \mathrm{cm}^{3}$. Portanto, algumas folhosas de baixa densidade podem ser consideradas para a aplicação em MLC, quando facilmente coláveis, uma vez que um estudo de Plaster et al. (2008), sobre o comportamento de juntas coladas da madeira serrada de Eucalipto sp., chegou a conclusão de que a densidade influenciou a adesão das juntas. Cabe mencionar os trabalhos correlatos de Teles et al. (2010), que confeccionaram vigas de MLC a partir de LouroVermelho (Sextonia rubra), de Terezo e Szücs (2010), que analisaram o desempenho de vigas de MLC fabricadas de Paricá (Schizolobium Amazonicum Huber ex. Ducke) obtido de floresta plantada, e de Cunha e Matos (2011) que avaliaram a rigidez de vigas estruturais de MLC coladas por meio de adesivo poliuretano.

A espécie utilizada neste trabalho foi o Cedrinho (Erisma uncinatum Warm.) que, em estudo prévio, apresentou os melhores resultados em relação a outras essências nativas para uso na fabricação de MLC
(ZANGIÁCOMO; LAHR, 2007), uma vez que, obtevese densidade igual a $0,62 \mathrm{~g} / \mathrm{cm}^{3}$, indicada para a produção de MLC, e compatibilidade com os adesivos. Vale ressaltar que, tão importante quanto à densidade, é a permeabilidade da espécie em relação aos adesivos disponíveis para a produção de MLC. De acordo com IPT (2009), a espécie nativa Cedrinho (Erisma uncinatum Warm.) é árvore da família Vochysiaceae e ocorre em toda a região amazônica, em especial no Estado do Amazonas. Apresenta permeabilidade que permite o tratamento do cerne e do alburno moderadamente fáceis de preservar em processos sob pressão e também é conhecida com outros nomes populares como bruteiro, cambará, cambará-rosa etc.

Neste estudo, objetivou-se determinar e relacionar o módulo de elasticidade dinâmico $\left(\mathrm{E}_{\mathrm{Mvt}}\right)$ com o módulo de elasticidade estático $\left(\mathrm{E}_{\mathrm{M}}\right)$, o módulo de ruptura $\left(\mathrm{f}_{\mathrm{M}}\right)$, a resistência à compressão $\left(\sigma_{\mathrm{cp}}\right)$ e o módulo de elasticidade paralelo $\left(\mathrm{E}_{\mathrm{cp}}\right)$, e avaliar o desempenho da espécie nativa Cedrinho (Erisma uncinatum Warm.) na construção de vigas de MLC produzidas a partir de lâminas sem emendas longitudinais.

\section{MATERIAL E MÉTODOS}

\subsection{Processo de confecção das vigas estruturais}

Para a confecção das vigas de MLC de dimensões nominais $6 \mathrm{~cm} \times 12 \mathrm{~cm} \times 300 \mathrm{~cm}$, foram utilizadas peças de madeira nativa da espécie Cedrinho (Erisma uncinatum Warm.), provenientes de floresta tropical certificada. Foram adquiridas tábuas de seção com dimensões nominais de 20 $\mathrm{mm} \times 300 \mathrm{~mm}$ e comprimentos que variaram entre $4000 \mathrm{e}$ $6000 \mathrm{~mm}$, no comércio madeireiro da região. Essas tábuas foram serradas em lâminas de dimensões nominais de 20 mm x $60 \mathrm{~mm}$ x $3000 \mathrm{~mm}$, totalizando 116 lâminas sem emendas longitudinais. Na confecção das vigas de MLC, foram utilizadas 96 lâminas de madeira nativa serrada com teor médio de umidade igual a $12 \%$. Na colagem das lâminas face a face, foram utilizados os adesivos bicomponentes Resorcina Fenol Formaldeído (RFF) e Poliuretano (PUR), sendo o primeiro conhecido pelo nome comercial de Cascophen e usual na produção de elementos estruturais de MLC, na indústria, e o segundo de cura a frio com tempo de utilização igual a 20 minutos e extraído à base da resina de mamona, desenvolvido e produzido por pesquisadores do Instituto de Química de São Carlos da Universidade de São Paulo (IQSC-USP).

Após a classificação visual, feita para a retirada das peças com defeitos, e mecânica para determinação dos $\mathrm{E}_{\mathrm{M}}$

Cerne, Lavras, v. 19, n. 3, p. 441-449, jul./set. 2013 
das lâminas, por ensaios de flexão estática, estas foram agrupadas em ordem decrescente de $\mathrm{E}_{\mathrm{M}}$ e separadas em dois lotes: um para montagem de 8 vigas com distribuição não aleatória de lâminas (NA), onde as lâminas com $\mathrm{E}_{\mathrm{M}}$ mais elevados foram dispostas nas regiões mais solicitadas da peça, e as de $E_{M}$ mais baixos foram colocadas nas regiões de menor solicitação; o outro para montagem de 8 vigas com distribuição aleatória (A) das lâminas, sem levar em conta a disposição do $\mathrm{E}_{\mathrm{M}}$ nas regiões mais e menos solicitadas da peça. Cada elemento estrutural foi formado por 6 lâminas sobrepostas, totalizando 16 vigas de MLC.

$\mathrm{O}$ consumo de adesivo foi de $450 \mathrm{~g} / \mathrm{m}^{2}$ para $\mathrm{RFF}$ e de $350 \mathrm{~g} / \mathrm{m}^{2}$ para PUR. Na união das lâminas, 8 vigas foram coladas com RFF, sendo 4 com intensidade de pressão igual a $0,8 \mathrm{MPa}$ e 4 com intensidade de pressão 1,2 MPa; as outras 8, juntas em grupo de 4, foram coladas com PUR, com as mesmas intensidades de pressão. $\mathrm{O}$ tempo de aplicação de pressão foi de 10 horas para ambos os adesivos. O tempo de cura das peças coladas foi de, no mínimo, 10 horas para o adesivo RFF e de 4 dias para o adesivo PUR. Após o período de cura, as vigas foram aparelhadas para ensaio.

\subsection{Ensaio de flexão estática}

Para a obtenção dos valores de $E_{M}$ e $f_{M}$ foi feito o ensaio de flexão estática, conforme a norma ASTM D 198, da American Society for Testing and Materials - ASTM (1997), com a configuração de viga simplesmente apoiada e carga concentrada na metade do vão livre. As vigas foram flexionadas em relação ao eixo de maior inércia.

As vigas foram apoiadas sobre roletes metálicos e, na metade do seu comprimento, aplicou-se força (P) até o deslocamento vertical $(\delta)$, no centro, atingir 14,1 $\mathrm{mm}$, estabelecido em função da flecha máxima (L/200) indicada pela NBR 7190, da ABNT (1997). Tal medida se fez com relógio comparador de resolução $0,01 \mathrm{~mm}$ e curso de $50 \mathrm{~mm}$.

Como a relação L/h (vão/altura) das vigas de MLC foi aproximadamente igual a $25, \mathrm{E}_{\mathrm{M}}$ e $\mathrm{f}_{\mathrm{M}}$ foram calculados empregando-se as Equações 1 e 2, respectivamente. $\mathrm{O} \mathrm{E}_{\mathrm{M}}$ foi calculado com base em um ponto do trecho linear do diagrama $\mathrm{P} \times \delta$, deslocamento vertical $(\delta)$ igual a $14,1 \mathrm{~mm}$, e o $\mathrm{f}_{\mathrm{M}}$ foi calculado com base na força máxima de ruptura $\mathrm{P}_{\text {rup }}$ das vigas de MLC, aplicada durante o ensaio de flexão estática, ambos conforme ASTM D 198, da ASTM (1997).

$\mathrm{E}_{\mathrm{M}}=\frac{\mathrm{P} \cdot \ell^{3}}{4 \cdot \delta \cdot \mathrm{b} \cdot \mathrm{h}^{3}}$
$f_{M}=\frac{3 \cdot P_{\text {rup }} \cdot \ell}{2 \cdot b \cdot h^{2}}$

sendo: $\mathrm{E}_{\mathrm{M}}$ o módulo de elasticidade $(\mathrm{MPa}) ; \mathrm{f}_{\mathrm{M}} \mathrm{o}$ módulo de ruptura $(\mathrm{MPa}) ; \mathrm{P}$ a força aplicada $(\mathrm{N}) ; \mathrm{P}_{\text {rup }}$ a carga de ruptura $(\mathrm{N}) ; \ell$ a distância entre apoios $(\mathrm{mm}) ; \delta$ o deslocamento vertical devido à força aplicada $(\mathrm{mm})$; b a largura da viga $(\mathrm{mm})$ e h a altura da viga $(\mathrm{mm})$.

\subsection{Ensaio de vibração transversal}

Experiências de Carreira et al. (2012) e Chui (1999) revelaram que o arranjo de ensaio mais confiável para caracterização das propriedades mecânicas de uma viga, por meio de ensaio de vibração transversal, é adotar a condição de contorno livre-livre. Os autores citados descrevem detalhes do procedimento deste ensaio em vigas de madeira serrada.

$\mathrm{O}$ procedimento utilizado para obter o $\mathrm{E}_{\mathrm{Mvt}}$, por meio de ensaio de vibração transversal de uma viga estrutural, foi determinar a primeira frequência natural do primeiro modo de vibrar na condição de contorno livre-livre, em seguida medir a massa e as dimensões. A partir da equação de movimento de Timoshenko (1938), obteve-se a Equação 3 para calcular o $\mathrm{E}_{\mathrm{Mvt}}$.

$\mathrm{E}_{\mathrm{Mvt}}=\frac{94,644 \cdot 10^{-5} \cdot \mathrm{f}_{1}^{2} \cdot \mathrm{m} \cdot \ell^{3}}{\mathrm{~b} \cdot \mathrm{h}^{3}}$

sendo: $\mathrm{E}_{\mathrm{Mvt}}$ o módulo de elasticidade $(\mathrm{MPa}) ; \mathrm{f}_{1}$ a frequência do primeiro modo de vibrar $(\mathrm{Hz})$; m a massa da viga $(\mathrm{kg})$; $\ell$ o comprimento da viga $(\mathrm{mm})$; b a largura da viga $(\mathrm{mm})$ e h a altura da viga ( $\mathrm{mm})$.

Nesse ensaio, com a condição de contorno livrelivre, as vigas foram suspensas por cordas de nylon, $2,5 \mathrm{~mm}$ de diâmetro, presas a duas molas de aço de baixa rigidez na extremidade superior, fixadas em um perfil metálico. $\mathrm{Na}$ outra extremidade, as cordas foram posicionadas nos pontos nodais $(0,224 \ell)$ do primeiro modo de vibrar, simulando a condição de contorno livre-livre e minorando a influência do sistema de suspensão nas frequências do primeiro modo, Figura 1.

Em uma das extremidades das vigas foi fixado um acelerômetro modelo ISOTRON 7254A-100, fabricado pela Endevco Corporation, com sensibilidade igual a $111,6 \mathrm{mV} / \mathrm{g}$. A excitação das vigas foi feita por meio de martelo de impacto modelo 8206-002, fabricado pela Brüel \& Kjcer, com sensibilidade igual a 2,3 mV/N. Os sinais de excitação (martelo) e resposta (acelerômetro)

Cerne, Lavras, v. 19, n. 3, p. 441-449, jul./set. 2013 


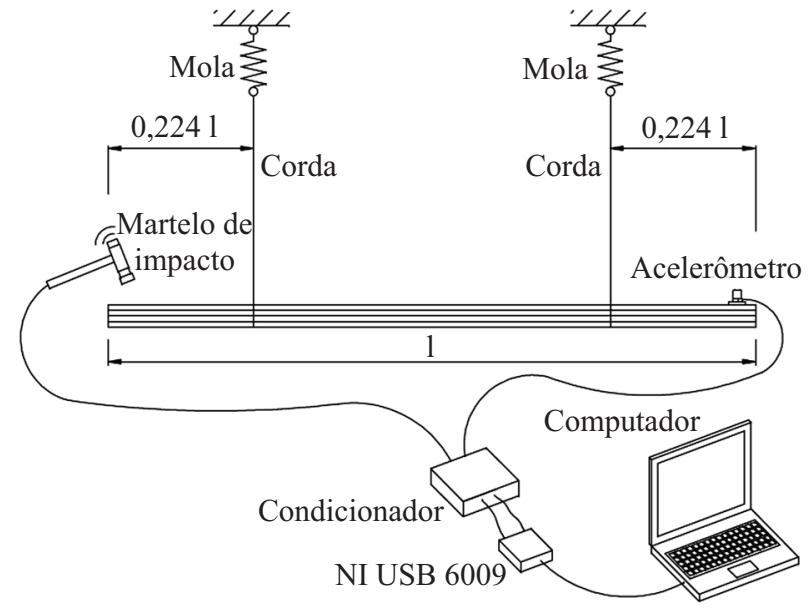

Figura 1 - Ensaio de vibração transversal de viga de MLC.

Figure 1 - Transverse vibration test of glulam beam.

foram amplificados e, na sequência, foi feita a conversão analógico-digital (ADC) por meio de uma placa, nesse caso módulo via porta USB modelo NI USB 6009, fabricada pela National Instruments. O programa de aquisição de dados e análise de sinais foi feito na linguagem de programação desenvolvida pela National Instruments chamada LabVIEW (Laboratory Virtual Instruments Engineering Workbench), linguagem gráfica de programação que usa ícones em vez de linhas de texto.

A partir da análise de sinais das Funções de Resposta em Frequência (FRF) das vigas de MLC, obtiveram-se os parâmetros modais (frequência e amortecimento) referentes ao primeiro modo de vibrar das vigas de MLC. Esses parâmetros modais foram identificados pelo método de ajuste modo a modo, chamado "peak-picking" (HE; FU, 2001), a partir da média de dez FRF experimentais para o primeiro modo de vibrar, conforme exemplo mostrado na Figura 2.

\subsection{Ensaios de corpos de prova retirados das vigas de MLC}

Após os ensaios para obter $\mathrm{E}_{\mathrm{Mvt}}, \mathrm{E}_{\mathrm{M}}$ e $\mathrm{f}_{\mathrm{M}}$ das vigas de MLC, foram retirados dois corpos de prova de cada viga de MLC, sendo um para ensaio de compressão paralela às fibras de madeira e outro para ensaio de delaminação, Figura 3, todos com a finalidade de avaliar a qualidade das vigas de MLC.

Os ensaios dos corpos de prova de compressão paralela às linhas de cola retirados das vigas de MLC foram feitos na Máquina Universal Servo-Hidráulica

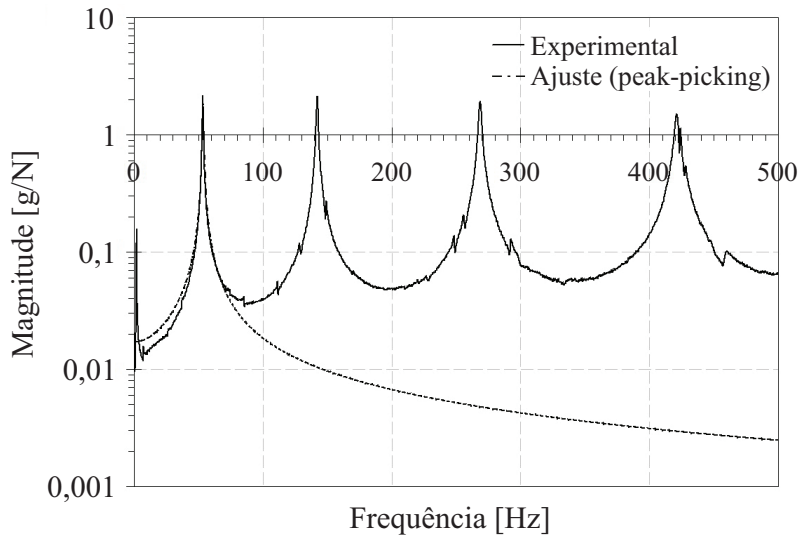

Figura 2 - FRF experimental obtida no ensaio dinâmico de viga de MLC.

Figure 2-Experimental FRF obtained in the dynamic test of glulam beam.

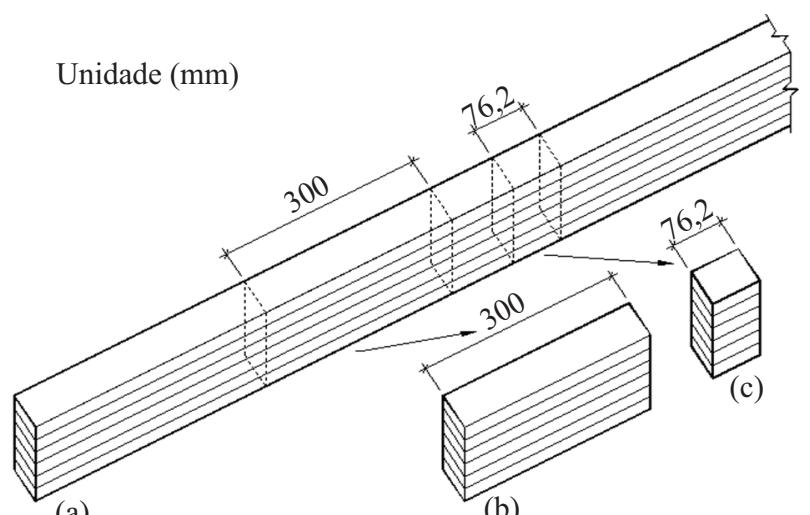

(a)

(b)

Figura 3 - (a) Viga de MLC, (b) corpo de prova de compressão paralela às linhas de cola e (c) corpo de prova de delaminação.

Figure 3 - (a) Glulam beam, (b) compression parallel to glue lines specimen and (c) lamination specimen.

modelo 8506/Custom, fabricada pela INSTRON, com capacidade para 2500 kN (ensaios dinâmicos) e 3000 kN (ensaios estáticos). Os ensaios foram realizados para se obter, simultaneamente, a resistência à compressão $\left(\sigma_{c p}\right)$ e o módulo de elasticidade paralelo $\left(\mathrm{E}_{\mathrm{cp}}\right)$ às linhas de cola, a partir das curvas tensão-deformação. A velocidade de carregamento imposta foi tomada igual a $0,008 \mathrm{~mm} / \mathrm{s}$, por meio de controle automático de deslocamento. Além desse controle, quatro transdutores de deslocamento registraram automaticamente, em cada uma das faces, os dados para uma base de medida igual a $100 \mathrm{~mm}$, compreendida na região central do corpo de prova, Figura 4.

Cerne, Lavras, v. 19, n. 3, p. 441-449, jul./set. 2013 


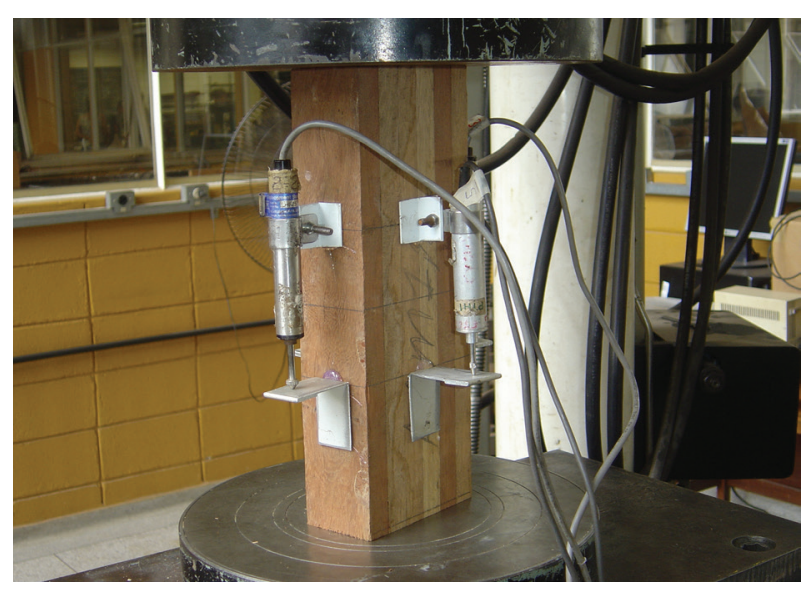

Figura 4 - Ensaio de corpo de prova de compressão paralela às linhas de cola.

Figure 4 - Specimen test of compression glue lines parallel.

O ensaio de delaminação foi feito conforme a norma AITC T110, da American Institute of Timber Construction - AITC (2007), que trata da avaliação de adesivos para uso estrutural de produtos de madeira expostos ao exterior. O ensaio consiste em colocar os corpos de prova dentro da autoclave, expondo a linha de cola a tensões, em decorrência do vácuo e pressão, em três ciclos de umedecimento e secagem, durante um período de 12 dias. Na primeira etapa, os corpos de prova, de seção igual à das vigas de MLC e $76,2 \mathrm{~mm}$ de comprimento, foram colocados na autoclave para a aplicação de vácuo de $75 \pm 10 \mathrm{kPa}$, durante 2 horas, depois é liberado o vácuo e aplica-se água com pressão de $540 \pm 20 \mathrm{kPa}$, durante 2 horas. Repetiu-se esse mesmo procedimento na segunda etapa, totalizando 8 horas de permanência na autoclave e, então, os corpos de prova foram retirados da autoclave e passaram por um período de secagem de 88 horas, ao ar livre, a uma temperatura de $28 \pm$ $2^{\circ} \mathrm{C}$, totalizando um período de 96 horas, no primeiro ciclo. No segundo e terceiro ciclos, foram repetidos os mesmo procedimentos do primeiro ciclo. Ao final do ensaio, previuse o comportamento da combinação da MLC exposta ao exterior, por meio da porcentagem de delaminação $\left(l_{l}\right)$, que é obtida nas duas faces de topo dos corpos de prova. Tal porcentagem é dada pelo comprimento de cada linha de cola em que ocorreu delaminação, dividido pelo comprimento total da linha de cola.

\subsection{Análise estatística}

Para análise estatística foram utilizados: a análise de regressão (linear simples), com a correspondente análise de variância; o teste de desvios (ou resíduos); o teste de normalidade de Shapiro-Wilks; e o teste de significância para o coeficiente de correlação. Com a análise de regressão foi possível relacionar a variável do ensaio de vibração transversal $\left(\mathrm{E}_{\mathrm{Mvv}}\right)$ às variáveis dos ensaios mecânicos $\left(\mathrm{E}_{\mathrm{M}}, \mathrm{f}_{\mathrm{M}}, \mathrm{E}_{\mathrm{cp}} \mathrm{e} \sigma_{\mathrm{cp}}\right)$. Com o teste dos desvios, foi possível verificar a existência (ou não) de tendenciosidade dos desvios (ou resíduos). Com o teste de normalidade de Shapiro-Wilkis foi possível verificar a normalidade (ou não) da distribuição dos desvios (ou resíduos).

\section{RESULTADOS E DISCUSSÃO}

$\mathrm{Na}$ Tabela 1, apresentam-se as resistências médias das trações (paralela e normal) às fibras e do cisalhamento paralelo às fibras para madeira Cedrinho (Erisma uncinatum Warm.). Os resultados apresentados são referentes à madeira maciça e, quando coladas, com os adesivos RFF e PUR. Tais valores foram obtidos por Zangiácomo e Lahr (2007) quando da realização de ensaios preliminares para buscar da espécie nativa alternativas dentre Envira Branca (Xylopia sp), Cambará (Moquinia polymorpha) e Castanheira (Bertholetia excelsa), a que melhor se adequava no emprego para produção de elementos estruturais de MLC.

Tabela 1 - Resistências do Cedrinho (Erisma uncinatum Warm.), conforme Zangiácomo e Lahr (2007).

Table 1 - Strenght of the Cedrinho (Erisma uncinatum Warm.), according to Zangiácomo e Lahr (2007).

\begin{tabular}{ccccc}
\hline Resistências (MPa) & Maciça & Pressão & RFF & PUR \\
\hline Cisalhamento & 8,37 & 0,8 & 8,13 & 8,13 \\
Tração normal & 2,57 & 0,8 & 2,21 & 2,15 \\
& & 0,8 & 33,47 & 37,48 \\
Tração paralela & \multirow{2}{*}{40,60} & 1,6 & 34,28 & 40,44 \\
\hline
\end{tabular}

Na Tabela 1, verifica-se que a resistência ao cisalhamento da madeira maciça foi apenas $2,9 \%$ superior ao cisalhamento da linha de cola para ambos os adesivos, (RFF) e (PUR), que foram utilizados na fabricação das vigas de MLC. Essa baixa diferença, levando-se em conta dois adesivos distintos, indica uma adesão eficiente nas vigas de MLC produzidas a partir da madeira tropical Cedrinho (Erisma uncinatum Warm.) e a densidade abaixo de $0,75 \mathrm{~kg} / \mathrm{m}^{3}$ é um dos fatores responsáveis que contribuem para essa eficiência, pois conforme Plaster et al. (2008) a densidade abaixo de $0,70 \mathrm{~kg} / \mathrm{m}^{3}$ havia influenciado a adesão das juntas na madeira serrada de Eucalipto $s p$.

Cerne, Lavras, v. 19, n. 3, p. 441-449, jul./set. 2013 
Na Tabela 2, são apresentados os valores referentes às dimensões $(\ell, \mathrm{b}$ e $\mathrm{h}$ - ver Figura 1$)$, massas $(\mathrm{m})$, frequências $\left(f_{1}\right)$, densidade aparente $(D A)$ e carga de ruptura $\left(\mathrm{P}_{\text {rup }}\right)$ das vigas de MLC.

$\mathrm{Na}$ Tabela 3, são mostrados os resultados dos ensaios realizados sob flexão estática e vibração transversal das vigas de MLC. Também são apresentados os valores de $\mathrm{E}_{\mathrm{M}}{ }^{*}$ obtidos por Zangiácomo e Lahr (2007). Tais valores de $E_{M}{ }^{*}$ foram obtidos por meio de ensaios de quatro pontos nas vigas de MLC, segundo a NBR 7190, da ABNT (1997) e a ASTM D 198, da ASTM (1997).

$\mathrm{Na}$ Tabela 4, são mostrados os resultados dos ensaios dos corpos de prova de compressão paralela às fibras e de delaminação da linha de cola das vigas de MLC.
Na Tabela 5 são apresentados os resultados do teste de normalidade de Shapiro-Wilks e a análise de variância (ANOVA) das variáveis.

Verificou-se que, a partir do teste dos desvios, não ocorreu tendenciosidade nem heterocedasticidade (variabilidade de variância) e o teste de normalidade de Shapiro-Wilk sugere distribuição normal dos desvios das variáveis, conforme apresentado na Tabela 5. Também observou-se que não existiu regressão para as variáveis $\mathrm{f}_{\mathrm{M}} \times \mathrm{E}_{\mathrm{Mvt}}$.

$\mathrm{Na}$ Tabela 6, são apresentadas as regressões lineares entre o módulo de elasticidade $\mathrm{E}_{\mathrm{Mvt}}$ e os módulos de elasticidade $E_{M}$ e $E_{c p}$, e a tensão de compressão $\sigma_{c p}$, indicadas juntamente com o respectivo coeficiente de correlação linear $\mathrm{R}^{2}$.

Tabela 2 - Dimensões e propriedades de flexão das vigas de MLC.

Table 2 - Size and bending properties of glulam beams.

\begin{tabular}{|c|c|c|c|c|c|c|c|}
\hline Vigas & $\ell(\mathrm{mm})$ & $\mathrm{b}(\mathrm{mm})$ & $\mathrm{h}(\mathrm{mm})$ & $\mathrm{m}(\mathrm{kg})$ & $\mathrm{f}_{1}(\mathrm{~Hz})$ & $\mathrm{DA}\left(\mathrm{g} / \mathrm{cm}^{3}\right)$ & $\mathrm{P}_{\text {rup }}(\mathrm{N})$ \\
\hline NA-1-C-0,8 & 2921 & 60,50 & 123,00 & 12,05 & 66,4 & 0,55 & 8434 \\
\hline NA-2-C-0,8 & 2911 & 61,25 & 122,25 & 12,25 & 67,1 & 0,56 & 8434 \\
\hline NA-1-C-1,2 & 2982 & 57,50 & 108,50 & 12,75 & 53,6 & 0,69 & 10777 \\
\hline NA-2-C-1,2 & 2978 & 57,50 & 112,75 & 12,30 & 52,2 & 0,64 & 13354 \\
\hline NA-1-M-0,8 & 2921 & 59,50 & 122,50 & 12,20 & 64,5 & 0,57 & 13823 \\
\hline NA-2-M- 0,8 & 2944 & 60,50 & 121,75 & 12,50 & 63,9 & 0,58 & 11714 \\
\hline NA-1-M-1,2 & 2981 & 56,75 & 109,00 & 12,90 & 55,7 & 0,70 & 13354 \\
\hline NA-2-M-1,2 & 2979 & 57,00 & 111,50 & 12,65 & 52,2 & 0,67 & 9137 \\
\hline Média & 2957 & 58,57 & 115,46 & 12,51 & 58,5 & 0,63 & 11513 \\
\hline DP & 30,84 & 1,82 & 6,43 & 0,27 & 6,46 & 0,06 & 2153,38 \\
\hline CV (\%) & 1,04 & 3,10 & 5,57 & 2,16 & 11,05 & 9,44 & 18,70 \\
\hline A-1-C- 0,8 & 2981 & 57,00 & 111,00 & 13,00 & 52,2 & 0,69 & 10777 \\
\hline A-2-C- 0,8 & 2982 & 56,50 & 111,25 & 12,50 & 55,4 & 0,67 & 14385 \\
\hline A-1-C-1,2 & 2981 & 57,00 & 111,25 & 12,50 & 53,6 & 0,66 & 7497 \\
\hline A-2-C-1,2 & 2980 & 56,50 & 109,50 & 12,65 & 50,4 & 0,69 & 8903 \\
\hline A-1-M-0,8 & 2981 & 56,75 & 109,50 & 12,75 & 51,0 & 0,69 & 11714 \\
\hline A-2-M-0,8 & 2981 & 56,50 & 109,25 & 13,05 & 51,3 & 0,71 & 11245 \\
\hline A-1-M-1,2 & 2982 & 57,00 & 109,75 & 12,75 & 47,8 & 0,68 & 7216 \\
\hline A-2-M-1,2 & 2979 & 57,00 & 109,00 & 12,90 & 49,6 & 0,70 & 8434 \\
\hline Média & 2981 & 56,78 & 110,06 & 12,76 & 51,4 & 0,69 & 10021 \\
\hline DP & 0,99 & 0,25 & 0,94 & 0,21 & 2,36 & 0,02 & 2449,13 \\
\hline CV (\%) & 0,03 & 0,44 & 0,86 & 1,65 & 4,58 & 2,33 & 24,44 \\
\hline
\end{tabular}

$\mathrm{Na}$ qual: NA significa não aleatória; A significa aleatória; 1 e 2 corresponde ao número das vigas; C é o adesivo resorcina fenol formaldeído (RFF); M é o adesivo poliuretano (PUR); 0,8 para pressão de colagem igual a 0,8 MPa e 1,2 para pressão de colagem igual a 1,2 $\mathrm{MPa}$.

Cerne, Lavras, v. 19, n. 3, p. 441-449, jul./set. 2013 
Tabela 3 - Propriedades de flexão das vigas de MLC.

Table 3 - Bending properties of glulam beams.

\begin{tabular}{ccccc}
\hline Vigas & $\begin{array}{c}\mathrm{E}_{\mathrm{M}}{ }^{*} \\
(\mathrm{MPa})\end{array}$ & $\begin{array}{c}\mathrm{E}_{\mathrm{M}} \\
(\mathrm{MPa})\end{array}$ & $\begin{array}{c}\mathrm{f}_{\mathrm{M}} \\
(\mathrm{MPa})\end{array}$ & $\begin{array}{c}\mathrm{E}_{\mathrm{Mvt}} \\
(\mathrm{MPa})\end{array}$ \\
\hline NA-1-C-0,8 & 11544 & 10987 & 39,3 & 11123 \\
NA-2-C-0,8 & 11966 & 11734 & 39,3 & 11520 \\
NA-1-C-1,2 & 11662 & 11919 & 67,8 & 12498 \\
NA-2-C-1,2 & 10017 & 9236 & 77,8 & 10159 \\
NA-1-M-0,8 & 10835 & 10961 & 65,9 & 10961 \\
NA-2-M-0,8 & 11247 & 11503 & 55,6 & 11301 \\
NA-1-M-1,2 & 12307 & 13465 & 84,4 & 13651 \\
NA-2-M-1,2 & 10836 & 10116 & 54,9 & 10909 \\
\hline Média & 11302 & 11240 & 60,6 & 11515 \\
DP & 729,63 & 1261,86 & 16,51 & 1084,85 \\
CV (\%) & 6,46 & 11,23 & 27,23 & 9,42 \\
\hline A-1-C-0,8 & 10612 & 10497 & 65,4 & 11386 \\
A-2-C-0,8 & 11752 & 11742 & 87,6 & 12372 \\
A-1-C-1,2 & 11312 & 10911 & 45,3 & 11454 \\
A-2-C-1,2 & 9869 & 9748 & 56,0 & 10830 \\
A-1-M-0,8 & 10256 & 10727 & 73,3 & 11143 \\
A-2-M-0,8 & 10506 & 11365 & 71,0 & 11673 \\
A-1-M-1,2 & 8713 & 9597 & 44,8 & 9687 \\
A-2-M-1,2 & 9757 & 10312 & 53,1 & 10753 \\
\hline Média & 9198 & 9435 & 58,2 & 9923 \\
DP & 3558,45 & 3600,80 & 18,18 & 3789,46 \\
CV (\%) & 38,69 & 38,17 & 31,24 & 38,19 \\
\hline & & & & \\
\hline
\end{tabular}

Tabela 4 - Propriedades mecânicas dos corpos de prova obtidos das vigas de MLC.

Table 4-Mechanical properties of samples obtained of glulam beams.

\begin{tabular}{ccccc}
\hline Vigas & $\mathrm{E}_{\mathrm{cp}}(\mathrm{MPa})$ & $\sigma_{\mathrm{cp}}(\mathrm{MPa})$ & $n\left(l_{c}\right)$ & $l_{l c D}(\%)$ \\
\hline NA-1-C-0,8 & 8459 & 36,8 & 9 & 7,39 \\
NA-2-C-0,8 & 9355 & 40,8 & 9 & 16,78 \\
NA-1-C-1,2 & 9945 & 45,1 & 10 & 0,00 \\
NA-2-C-1,2 & 7533 & 38,1 & 10 & 6,72 \\
NA-1-M-0,8 & 8417 & 36,9 & 10 & 0,00 \\
NA-2-M-0,8 & 9347 & 38,7 & 10 & 3,25 \\
NA-1-M-1,2 & 9905 & 45,8 & 10 & 3,27 \\
NA-2-M-1,2 & 8939 & 43,2 & 10 & 0,00 \\
\hline Média & 8987 & 40,7 & - & - \\
DP & 823,46 & 3,63 & - & - \\
CV $(\%)$ & 9,16 & 8,91 & - & - \\
\hline A-1-C-0,8 & 11073 & 46,6 & 8 & 7,74 \\
A-2-C-0,8 & 10152 & 46,0 & 10 & 13,48 \\
A-1-C-1,2 & 8630 & 41,7 & 10 & 3,48 \\
A-2-C-1,2 & 7981 & 39,6 & 7 & 31,91 \\
A-1-M-0,8 & 9786 & 45,6 & 10 & 5,89 \\
A-2-M-0,8 & 10257 & 49,2 & 20 & 12,34 \\
A-1-M-1,2 & 8586 & 41,3 & 10 & 6,14 \\
A-2-M-1,2 & 7965 & 42,0 & 10 & 0,85 \\
\hline Média & 8271 & 40,1 & - & - \\
DP & 3284,35 & 12,10 & - & - \\
CV $(\%)$ & 39,71 & 30,16 & - & - \\
\hline
\end{tabular}

Tabela 5 - Resultados do teste de Shapiro-Wilk e ANOVA.

Table 5-Results of Shapiro-Wilk and ANOVA tests.

\begin{tabular}{cccc}
\hline Variáveis & Valor-p & ANOVA & Regressão \\
\hline $\mathrm{E}_{\mathrm{M}} \times \mathrm{E}_{\mathrm{Mvt}}$ & $0,704>0,05$ & $\mathrm{~F}_{\text {calculado }}=85,36>\mathrm{F}_{\text {tabelado }}=\mathrm{F}_{0,05 ; 1 ; 14}=4,60$ & Sim \\
$\mathrm{f}_{\mathrm{M}} \times \mathrm{E}_{\mathrm{Mvt}}$ & $0,776>0,05$ & $\mathrm{~F}_{\text {calculado }}=4,44<\mathrm{F}_{\text {tabelado }}=\mathrm{F}_{0,05 ; 1 ; 14}=4,60$ & Não \\
$\mathrm{E}_{\mathrm{cp}} \times \mathrm{E}_{\mathrm{Mvt}}$ & $0,087>0,05$ & $\mathrm{~F}_{\text {calculado }}=5,42>\mathrm{F}_{\text {tabelado }}=\mathrm{F}_{0,05 ; 1 ; 14}=4,60$ & Sim \\
$\sigma_{\mathrm{cp}} \times \mathrm{E}_{\mathrm{Mvt}}$ & $0,927>0,05$ & $\mathrm{~F}_{\text {calculado }}=9,01>\mathrm{F}_{\text {tabelado }}=\mathrm{F}_{0,05 ; 1 ; 14}=4,60$ & Sim \\
\hline
\end{tabular}

A partir da determinação das propriedades apresentadas nas Tabelas 3 e $4, \mathrm{E}_{\mathrm{Mvt}}, \mathrm{E}_{\mathrm{M}}, \mathrm{E}_{\mathrm{M}}{ }^{*}, \mathrm{f}_{\mathrm{M}}, \mathrm{E}_{\mathrm{cp}} \mathrm{e}$ $\sigma_{\mathrm{cp}}$, observa-se que os valores médios obtidos nas vigas de MLC produzidas com distribuição não aleatória das lâminas, tiveram ganhos de $13,8 \%, 16,1 \%, 18,6 \%, 4,0 \%$, $8,0 \%$ e $1,5 \%$, respectivamente, do que naquelas produzidas com distribuição aleatória das lâminas. Conforme Cunha e Matos (2011), quando avaliaram a rigidez de vigas de MLC produzidas com distribuição de lâminas aleatórias e não aleatórias, também constataram que houve um ganho médio de rigidez da ordem $6,6 \%$ pela distribuição não aleatória.

Cerne, Lavras, v. 19, n. 3, p. 441-449, jul./set. 2013 
Tabela 6 - Modelos de regressão linear $(\mathrm{y}=\mathrm{ax}+\mathrm{b})$ para estimativa das variáveis.

Table 6 - Linear regression models $(y=a x+b)$ to estimating of the variables.

\begin{tabular}{ccccc}
\hline $\mathrm{y}(\mathrm{MPa})$ & $\mathrm{x}(\mathrm{MPa})$ & $\mathrm{a}$ & $\mathrm{b}(\mathrm{MPa})$ & $\mathrm{R}^{2}$ \\
\hline $\mathrm{E}_{\mathrm{M}}$ & $\mathrm{E}_{\mathrm{Mvt}}$ & 1,044 & $-907,22$ & $0,86^{* *}$ \\
$\mathrm{E}_{\mathrm{cp}}$ & $\mathrm{E}_{\mathrm{Mvt}}$ & 0,663 & 1627,6 & $0,39^{* *}$ \\
$\sigma_{\mathrm{cp}}$ & $\mathrm{E}_{\mathrm{Mvt}}$ & 0,002 & 18,17 & $0,28^{\text {n.s. }}$ \\
\hline
\end{tabular}

Na qual: ${ }^{* *}$ Significativo ao nível de $1 \%$ de probabilidade $(\mathrm{P}<$

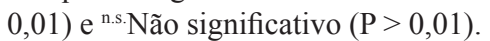

Os módulos de elasticidade obtido por meio do ensaio de vibração transversal livre $\mathrm{E}_{\mathrm{Mvt}}$ foram $2,4 \% \mathrm{e}$ $4,9 \%$, Tabela 3, maior do que o módulo de elasticidade calculado por meio do ensaio de flexão estática $\mathrm{E}_{\mathrm{M}}$, nas vigas de MLC com distribuição não aleatória e aleatória das lâminas, respectivamente. O comportamento dessas propriedades confirma o que os autores Mochan et al. (2009) haviam concluído, isto é, os módulos de elasticidade obtidos a partir de ensaios dinâmicos apresentam valores maiores que os ensaios de flexão estática.

\section{CONCLUSÕES}

Constatou-se, na presente pesquisa, que as rupturas de todas as vigas de MLC ocorreram por tração no ensaio de flexão estática.

$\mathrm{O} \mathrm{E}_{\mathrm{Mvt}}$ apresentou relação funcional com $\mathrm{E}_{\mathrm{M}}$, com valor de $\mathrm{R}^{2}$, próximo de 1,0 e correlação significativa (Tabela 6). Entretanto, observou-se que não existiu regressão entre $\mathrm{f}_{\mathrm{M}} \times \mathrm{E}_{\mathrm{Mvt}}$ (Tabela 5), não sendo possível ajustar uma equação adequada para representar a relação entre essas duas variáveis. Embora tenha sido possível ajustar as duas equações para expressar a relação entre $\mathrm{E}_{c p} \times \mathrm{E}_{\mathrm{Mvt}}$ e $\sigma_{c p} \times \mathrm{E}_{\mathrm{Mvt}}$ e foram obtidos valores de $\mathrm{R}^{2}$ distantes de 1,0 que apresentam correlação significativa e não significativa, respectivamente (Tabela 6). Assim sendo, pode-se afirmar que o $\mathrm{E}_{\mathrm{Mvt}}$ é um bom estimador do $\mathrm{E}_{\mathrm{M}}$, porém recomenda-se que não deve ser utilizado para fazer estimativas do $\mathrm{E}_{\mathrm{cp}}$ e $\sigma_{\mathrm{cp}}$.

A partir dos resultados dos ensaios dos corpos de prova de compressão paralela, observou-se que a resistência média $\sigma_{\mathrm{cp}}$ das vigas de MLC ficou próxima daquela citada para a madeira serrada dessa espécie, conforme IPT (2009). O módulo de elasticidade $\mathrm{E}_{\mathrm{cp}}$ corresponde aproximadamente a $83,7 \%$ do $\mathrm{E}_{\mathrm{M}}$, indicando uma relação próxima daquela recomendada na NBR 7190, da ABNT (1997), para madeiras serradas. O adesivo PUR teve melhor desempenho no ensaio de delaminação do que o adesivo RFF, conforme observado nos resultados da Tabela 4. A delaminação ficou 2,76 vezes menor em média, isto é, 3,97\% para 90 linhas de PUR e 10,94\% para 73 linhas de RFF.

A espécie de madeira tropical Cedrinho (Erisma uncinatum Warm.) pode ser utilizada na fabricação de vigas de MLC, tomando as condições necessárias na produção, conforme prescrita nas normas internacionais que tratam desse tema. A propriedade mecânica de compressão paralela às fibras $\sigma_{\mathrm{cp}}$ apresentada na Tabela 4 , obtidas em corpos de prova estruturais, permite classificar a espécie de madeira tropical Cedrinho (Erisma uncinatum Warm.) na classe de resistência C30 das folhosas segundo a NBR 7190, da ABNT (1997).

\section{AGRADECIMENTOS}

Agradecimentos à Fundação de Amparo à Pesquisa do Estado de São Paulo (FAPESP), Coordenação de Aperfeiçoamento de Pessoal de Nível Superior (CAPES) e Conselho Nacional de Desenvolvimento Científico e Tecnológico (CNPq) pelo aporte financeiro e ao Laboratório de Madeiras e de Estruturas de Madeira (LaMEM) por todo o suporte despendido na fabricação e ensaios das vigas de MLC.

\section{REFERÊNCIAS}

AMERICAN INSTITUTE OF TIMBER CONSTRUCTION. T110: test methods for structural glued laminated timber. Colorado, 2007.

AMERICAN SOCIETY FOR TESTING AND MATERIALS. D198: standard test methods of static tests of lumber in structural sizes. Philadelphia, 1997.

ASSOCIAÇÃO BRASILEIRA DE NORMAS TÉCNICAS. NBR 7190: projeto de estruturas de madeira. Rio de Janeiro, 1997.

BODIG, J.; JAYNE, B. A. Mechanics of wood and wood composites. New York: V. N. Reinhold, 1993.

CARREIRA, M. R.; SEGUNDINHO, P. G. A.; ROCCO

LAHR, F. A.; DIAS, A. A.; CALIL JUNIOR, C. Bending stiffness evaluation of Teca and Guajará lumber through tests of transverse and longitudinal vibration. Acta Scientiarum. Technology, Maringá, v. 34, n. 1, p. 27-32, Jan./Mar. 2012.

Cerne, Lavras, v. 19, n. 3, p. 441-449, jul./set. 2013 
CHUI, Y. H.; BARCLAY, D. W.; COOPER, P. A. Evaluation of wood poles using a free vibration technique. Journal of Testing and Evaluation, West Conshohocken, v. 27, n. 3, p. 191-195, May 1999.

CUNHA, A. B.; MATOS, J. L. M. Avaliação da rigidez de vigas estruturais de madeira laminada colada unidas por adesivo poliuretano. Cerne, Lavras, v. 17, n. 4, p. 593-600, out./dez. 2011.

HE, J.; FU, Z. F. Modal analysis. Oxford: ButterworthHeinemann, 2001.

INSTITUTO DE PESQUISAS TECNOLÓGICAS DO ESTADO DE SÃO PAULO. Madeira: uso sustentável na construção civil. São Paulo, 2009.

MOCHAN, S.; CONNOLLY, T.; MOORE, J. Using acoustic tools in forestry and the wood supply chain. Edinburgh: Crown, 2009.

PFEIL, W. E.; PFEIL, M. Estruturas de madeira. 6. ed. Rio de Janeiro: LTC, 2003.
PLASTER, O. B.; OLIVEIRA, J. T. S.; ABRAHÃO, C. P.; BRAZ, R. L. Comportamento de juntas coladas da madeira serrada de Eucalyptus sp. Cerne, Lavras, v. 14, n. 3, p. 251258, jul./set. 2008 .

TELES, R. F.; MENEZZI, C. H. S.; SOUZA, M. R.; SOUZA, F. Effect of nondestructive testing of laminations on the bending properties of glulam beams made from lourovermelho (Sextonia rubra). Cerne, Lavras, v. 16, n. 1, p. 77-85, jan./mar. 2010.

TEREZO, R. F.; SZÜCS, C. A. Análise de desempenho de vigas em madeira-laminada-colada de parica (Schizolobium amazonicum Huber ex. Ducke). Scientia Forestalis, Piracicaba, v. 38, n. 87, p. 471-480, set. 2010.

TIMOSHENKO, S. Vibration problems in engineering. $2^{\text {nd }} \mathrm{ed}$. New York: J. Wiley, 1938.

ZANGIÁCOMO, A. L.; LAHR, F. A. R. Emprego de espécies tropicais alternativas na produção de elementos estruturais de madeira laminada colada. Cadernos de Engenharia de Estruturas, São Carlos, v. 9, n. 40, p. 103-131, 2007.

Recebido: 10 de março de 2011; aceito: 28 de fevereiro de 2013.

Cerne, Lavras, v. 19, n. 3, p. 441-449, jul./set. 2013 
